\title{
Benchtop NMR Spectroscopy and Spectral Analysis of the cis- and trans-stilbene Products of the Wittig Reaction.
}

Mark Edgar $^{\star} \dagger$, Benita C. Percival ${ }^{\ddagger}$, Miles Gibson $^{\ddagger}$, Jinit Masania ${ }^{\ddagger}$, Ken Beresford ${ }^{\ddagger}$, Philippe B. Wilson ${ }^{\ddagger}$, Martin Grootveld

$5 \dagger$ School of Science, Loughborough University, Epinal Way, Loughborough, LE11 3TU, UK.

$\ddagger$ Leicester School of Pharmacy, De Montfort University, The Gateway, Leicester, LE1 9BH, UK.

Dedicated to Professor Raymond J. Abraham on his $86^{\text {th }}$ birthday (November 2019).

\section{ABSTRACT}

Benchtop NMR spectrometers are now becoming more widely employed in university teaching laboratories. These low-field instruments are increasingly used in reaction monitoring and product purity applications. NMR spectra obtained using these spectrometers $(40-80 \mathrm{MHz})$ tend to suffer from significant overlap of signals when compared to those obtained at $300-400 \mathrm{MHz}$ or above, and therefore some reactions may be less suited to analysis using such benchtop systems. Whilst some reactions can be modified to make them more amenable to analysis on low-field benchtop spectrometers, the fact remains that many common undergraduate laboratory chemistry reactions remain as a stalwart of the university education system. Therefore, there is currently a major requirement for benchtop NMR analysis to improve in order to facilitate student understanding. Herein, it is demonstrated a combination of spectral analysis and simulation at low-fields (40-80 $\mathrm{MHz}$ ) that allows the fine structure of second-order effects and overlapping spectra to be deduced, enabling an improved understanding of the low-field benchtop NMR technique within undergraduate student cohorts. The evolution of well-resolved and distinct multiplets at $400 \mathrm{MHz}$ to complex, overlapping multiplets at $40-80 \mathrm{MHz}$ also serves as a useful guide for laboratory demonstrators and academic staff when explaining the advantages of such benchtop systems. The Wittig reaction has been a standard reaction practical session in many university teaching laboratories since the 1980s, the products of which are a mixture of cis- and trans-stilbenes. This reaction serves as an ideal example of how benchtop NMR and analysis can support chemistry teaching laboratories. 


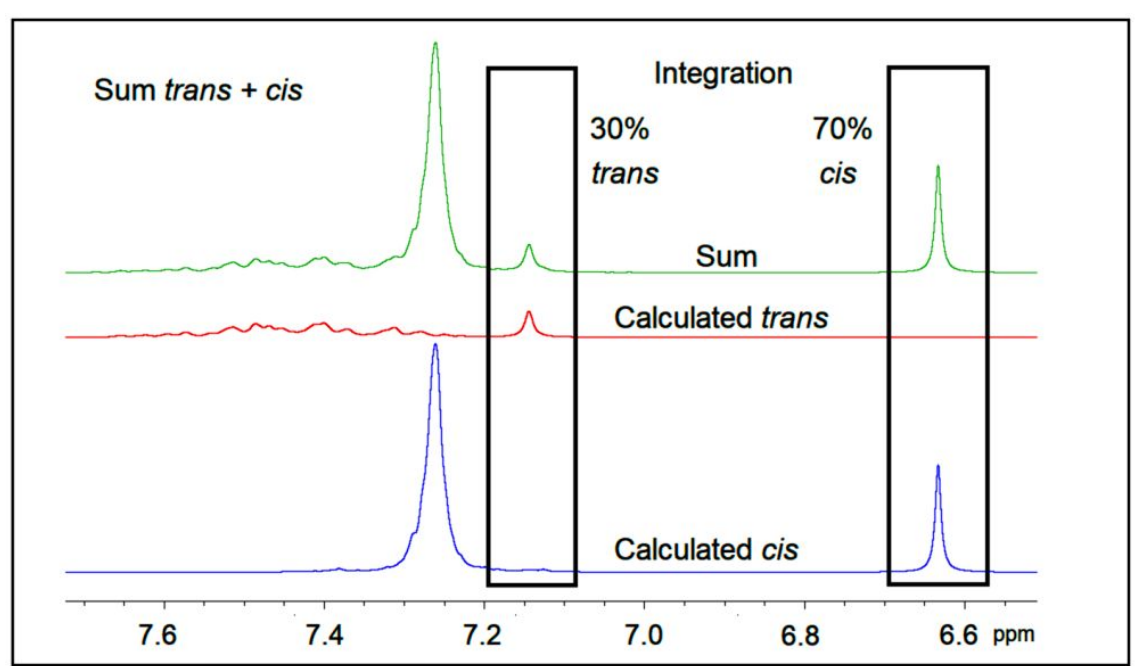

\section{KEYWORDS}

Second-Year Undergraduate, Laboratory Instruction, Organic Chemistry, Analysis, Inquiry-

Based/Discovery Learning, NMR Spectroscopy, conformational analysis

\section{INTRODUCTION}

The Wittig reaction ${ }^{1}$ was first published in 1953 , and this synthetic route has since become a significant economic and educational success, earning Wittig a Nobel prize in Chemistry in 1979. 40 In 1973, Markl and $\mathrm{Merz}^{2}$ reported the simultaneous preparation of cis- and trans-stilbenes from the Wittig condensation of benzaldehyde with benzyltriphenylphosphonium chloride (Scheme 1). This method was eminently applicable to undergraduate teaching laboratories and has been widely adopted in this context globally. ${ }^{3}$

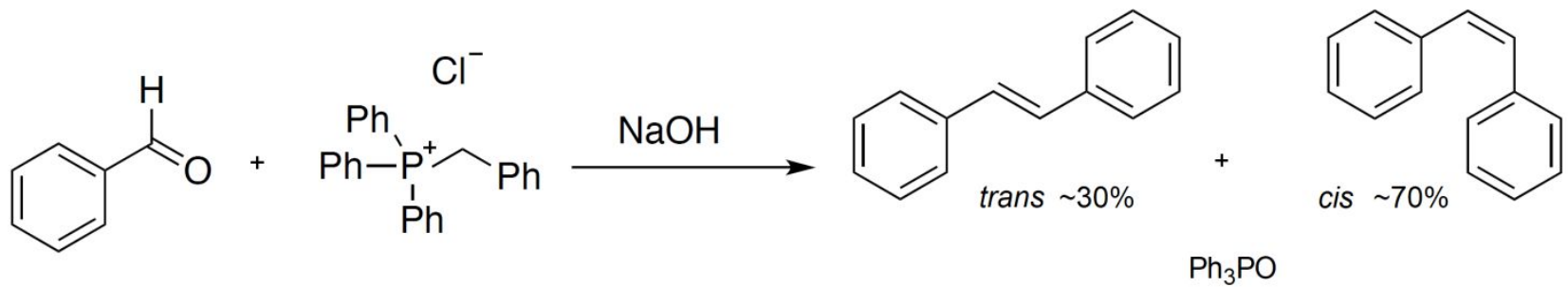

Scheme 1. Reaction of benzaldehyde with benzyltriphenylphosphonium chloride to form a mixture of cis-stilbene (major product), transstilbene (minor product) and by-product of triphenylphosphine oxide.

In principle, it is possible to separate the cis- and trans- stilbene regioisomers for their analytical characterization using techniques such as melting point, FTIR spectroscopy or electronic 

operating frequencies) ${ }^{4}$ is, of course, a significant consideration since these generally range between $\$ 100-300 \mathrm{~K}$, and, while these instruments may be financed as a research instrument on which undergraduate teaching time can be hired on an hourly rate basis, the full-economic cost per laboratory experiment and per student can be substantial. Currently, many novel applications are being explored for NMR spectroscopy, particularly with regard to the miniaturization of 'stateof-the-art' rapid analytical monitoring technologies. $., 6,7,8$ Indeed, many universities have now invested in low-field, benchtop NMR spectrometers and utilize them in chemistry undergraduate teaching experiments ${ }^{9,10,11}$ in order to determine reaction progress and product purities, for example. The value of the student experience that comes with direct access to a benchtop NMR instrument, however, must be considered when the purchase price is discussed; the cost of these facilities lies between $\$ 20-70 \mathrm{~K}$. Also, tangible and intangible savings of staff time and sample transport to high-field NMR instruments available support a stronger financial case to invest in benchtop NMR systems within the teaching laboratory. Herein, the advantages offered to a potentially wide range of undergraduate laboratory experiments by the application of benchtop NMR spectrometers is highlighted, and the benefits of supporting low-field spectral computations in tandem.

Few undergraduates have access to 'hands-on' training on large, high-field NMR spectrometers, with many analyses taking place as a remote service with the students receiving their NMR spectrum and associated data, or even a generic handout containing this information recorded some years prior. Unfortunately, this process, which disconnects key linkages between students and their institutional NMR spectrometer(s), strongly impacts on the educational value of such laboratory classes, i.e. pedagogically-important synchronous connections between NMR lecture materials and the on-site practical assessment of reaction products using this technique are 
80 broken (Figure 1). Therefore, this represents one of the most compelling reasons for inclusion of benchtop NMR spectrometers within undergraduate laboratories, in order to allow students to have a 'hands-on', real-time access to a means of analysis which provides substantial information on their analyte samples during laboratory classes. Recent advances in pedagogical analytical chemistry $^{12}$ have highlighted the importance of pooling novel and well-established teaching techniques to a course where the practical and theoretical components are intrinsically interlinked.

Several universities have had success using benchtop NMR spectrometers at an operating frequency of $45 \mathrm{MHz}$, and these low-field systems have been used to analyse Fischer esterification products, ${ }^{13}$ and the free radical-mediated bromination of ethylbenzene ${ }^{14}$. Moreover, the portability of these systems also permits them to be used in university-high school partnership program in the $\mathrm{USA}^{15}$, and university outreach strategies in the UK. ${ }^{16}$

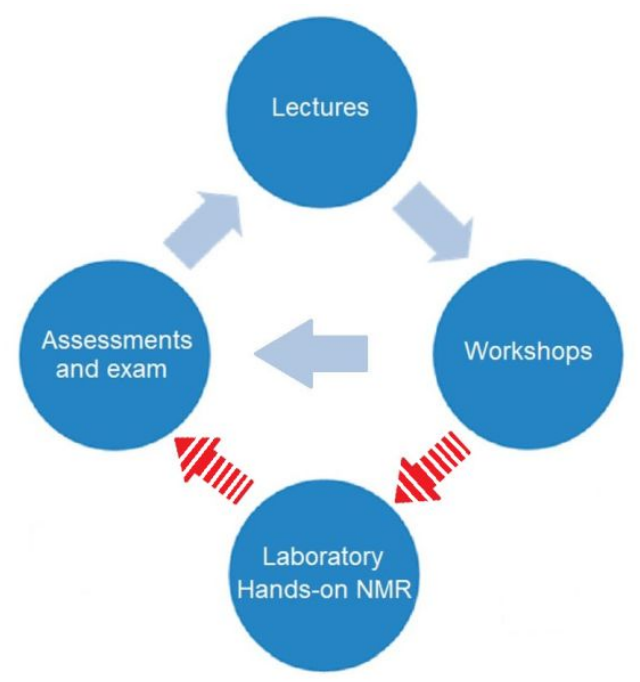

Figure 1. The disconnect in pedagogy (shown in red) caused by the removal of 'hands-on' NMR spectroscopy in teaching laboratories during a typical higher education institute academic year.

The teaching of NMR theory, that is offered at undergraduate level is focused on high-field magnets (300-400 MHz and beyond), however, the rules and principles do not strictly apply to lowfield benchtop NMR spectrometers in the same manner. ${ }^{17,18}$ In recent decades, many NMR courses and text books have evolved to the point where low-field NMR magnets are no longer mentioned, and the influence of first- and second-order effects are no longer covered in significant detail. The term "roofing" for the slight distortion away from the classic 1:1 1:2:1 and 1:3:3:1 Pascal's triangle 
intensities of simple multiplet resonances is insufficient to analyze a closely coupled ABX spinsystem. Hence, both undergraduates and university academic staff should develop the ability to appreciate modifications to the appearance of NMR spectra as a function of magnetic field strength.

As early as the middle of the $20^{\text {th }}$ century it was reasoned that the direct (through-space) dipole-dipole coupling between two hydrogen nuclei would average to zero in view of random isotropic motion in the liquid state which is indeed correct $(\mathrm{J}$-coupling tensor $3 \times 3$ matrix averages to zero). However, small couplings of a few $\mathrm{Hz}$ in magnitude were routinely observed. This was the subject of much debate but rationalized by the influence of the bonding electrons between the hydrogen atoms ( $\mathrm{H}-\mathrm{C}-\mathrm{H}$ or $\mathrm{H}-\mathrm{C}-\mathrm{C}-\mathrm{H}$ etc $)$ and is therefore referred to as the indirect (through-bond) dipole-dipole coupling. This interaction can be defined as the average of the diagonal of the elements of the $\mathrm{J}$-coupling tensor, which is scalar, indicating that the isotropic component of the $\mathrm{J}$ coupling Hamiltonian is independent of molecular motion. Now more commonly referred to as the $\mathrm{J}$-coupling in undergraduate chemistry lectures and text books, the size of this interaction between neighboring hydrogen atoms is the same at $40 \mathrm{MHz}$ as at $400 \mathrm{MHz}$; it is field independent, thus a typical ${ }^{3} \mathrm{~J}_{\mathrm{HH}}$ coupling in an aromatic group will correspond to $7.7 \mathrm{~Hz}$ at $40 \mathrm{MHz}$ as well as at 400 $\mathrm{MHz} .{ }^{19}$ As the magnitude of the $\mathrm{J}$-coupling remains the same size in different magnetic fields, this has significant influence on the appearance of NMR spectra recorded at different magnetic fields since the chemical shifts involved are field independent on the ppm scale, but are field dependent on the $\mathrm{Hz}$ scale . Consequently, by inspection, a doublet may appear to be "larger" at $40 \mathrm{MHz}(40$ $\mathrm{Hz}$ per ppm) than at $400 \mathrm{MHz}(400 \mathrm{~Hz}$ per ppm). This, in turn, leads on to the second-order nature of the spectra being more pronounced at low-field than high-field, in which a doublet can appear to be distorted and no longer adhere to a 1:1 intensity ratio. This distortion arises from the quantized energy levels that exist for the spin-system and the transition probabilities between each level therein. Undergraduates are taught that allowed transitions by established selection rules appear as resonances in the spectra, whereas forbidden transitions by the selection rules do not appear to be present therein. Thus, for a first-order spectrum both transitions that create a doublet are equally probable, hence producing the 1:1 doublet intensity ratio $\left(\mathrm{H}_{\mathrm{A}} \mathrm{H}_{\mathrm{X}}\right)$. When the chemical shift difference between the coupling pair of hydrogens (in $\mathrm{Hz}$ ) approaches approximately 10 times the $\mathrm{J}$ coupling between them, one of the transitions becomes less probable (less allowed), leading to a distorted doublet as indicated by the term "roofing" $\left(\mathrm{H}_{\mathrm{A}} \mathrm{H}_{\mathrm{B}}\right)$. For a strongly second-order system in 
which the chemical shift difference between the coupling pair of hydrogen nuclei (in $\mathrm{Hz}$ ) approaches approximately 5 times the $\mathrm{J}$-coupling between them, then the transition probability decreases significantly for one signal in the doublet, leading to a steeply roofed doublet $\left(\mathrm{H}_{\mathrm{A}} \mathrm{H}_{\mathrm{A}^{\prime}}\right)$. The natural end point occurs when the chemical shifts of the two hydrogen nuclei are equivalent i.e. they have the same chemical shift value, and in this model the transition probability of one signal in the doublet is zero, therefore giving rise to a signal that appears to be a singlet.

Herein, the high- and low-field NMR spectra of cis- and trans-stilbenes serve as an illustrative example to highlight these changes. In particular the computationally-simulated spectra decrementing from $400 \rightarrow 300 \rightarrow 200 \rightarrow 80 \rightarrow 60 \rightarrow 40 \mathrm{MHz}$, allows students to follow the evolution of multiplets from prominent and clearly distinct signals at the higher operating frequencies to those affected by lower resolution, with an increasing level of spectral overlap and significant second-order effects.

\section{CHEMICAL SHIFTS AND COUPLING CONSTANTS.}

Analysis of NMR multiplets to extract chemical shift and coupling constant values has been a central part of NMR spectroscopy since the 1950 s, ${ }^{20,21}$ when the $\mathrm{AA}^{\prime} \mathrm{BB}^{\prime}$ spin-system ${ }^{22}$ of thiophene, ${ }^{23}$ furan and pyrrole, ${ }^{24}$ and substituted fluoro-aromatics ${ }^{25}$ were recorded at 30, 40 and $60 \mathrm{MHz}$, and analyzed using pencil and paper calculations. Since that time, detailed theoretical ${ }^{26,27,28}$ and computational progress has vastly improved our solutions to such NMR problems. This resulted in the employment of computational methods such as Laocoon, ${ }^{29}$ and PANIC, ${ }^{30}$ and in more recent decades, by graphic-based calculations such as those featured in WinDNMR, ${ }^{31}$ Louiville calculations employing the experimental pulse-programs within NMR-SIM, ${ }^{32}$ iterative methods such as SpinWorks, ${ }^{33,34}$ and line-shape algorithm approaches, i.e. ANATOLIA in $2018,{ }^{35}$ such that it has never been easier to analyze experimental NMR spectra. In addition, the theoretical chemistry community has developed spectral prediction routines for common electronic structure codes such as Gaussian, ${ }^{36}$ allowing for the calculation of NMR shielding tensors and coupling from $a b$ initio methodologies as well as semi-empirical methods..$^{37,38}$

Mnova $^{39}$ is a popular suit of commercial software that can process, predict and analyze NMR spectra from all NMR vendors. One module allows extraction of first-order coupling constants but 
does not allow iterative analysis of second-order spin-systems. The chemical shift and coupling data extracted from highly second-order spectra using other methods can be input manually to generate simulated spectra. Spin Works is a popular spectral analysis program that employs an assign-iterate method to optimize spectral parameters, and works very well, however the assign process can be slow and time consuming even when the "automatic assign" feature is employed. Thus, multiple attempts to extract parameters can be time consuming, especially when input parameters produce a calculated spectrum that is very different to the experimental spectrum. Win-D-NMR is a graphical program that used chemical shift and coupling values to generate a spectrum, a significant advantage of this program is that a chemical shift and/or coupling constant value can be incremented with the resulting spectrum updated in real time, which allows an intuitive visual comparison to be made regarding the influence of parameters on the appearance of the spectrum. Bruker TopSpin4.0 is free for academic use and contains a line-shape analysis module, DAISY ${ }^{40}$ that can analyze first-order and some second-order spin-systems. TopSpin can import experimental data sets from benchtop spectrometers (JCAMP-DX), and from JEOL and Varian spectrometers ready for analysis using ANATOLIA.

It is prudent to consider chemical shift and scalar coupling constant values at an operating frequency of $400 \mathrm{MHz}$ before moving on to spectra recorded at only $60 \mathrm{MHz}$. The literature values for the $\mathrm{AA}^{\prime} \mathrm{BB}^{\prime} \mathrm{C}$ spin-system of the aromatic protons in stilbene are an excellent starting point for analysis, and the SpinWorks module ${ }^{30,31}$ and ANATOLIA generated excellent fits to the $400 \mathrm{MHz}{ }^{1} \mathrm{H}$ NMR spectra of the trans-regioisomer, which displayed small second-order effects within the clearly-defined doublets and triplets. However, the ${ }^{1} \mathrm{H}$ NMR spectrum of cis-stilbene was more complex, since the doublet and triplet patterns overlap significantly, even at an operating frequency of $400 \mathrm{MHz}$, and the second-order effects are significant $(\Delta \delta / \mathrm{J}<5)$ for all coupling nuclei. In this instance, the iterative line-shape analysis program ANATOLIA proved to be more user friendly and a faster analysis method than the traditional assign-iterate methods and achieved an excellent match with experimental results. The results are shown in Table 1 and 2

Table 1. Chemical Shift and Coupling Constant values for cis- and transstilbenes.

Cis-stilbene 


$\begin{array}{ccc}\delta A=\delta A^{\prime} & 7.285 \mathrm{ppm} & 7.550 \mathrm{ppm} \\ \delta B=\delta B^{\prime} & 7.259 \mathrm{ppm} & 7.391 \mathrm{ppm} \\ \delta C & 7.225 \mathrm{ppm} & 7.292 \mathrm{ppm} \\ \delta D & 6.633 \mathrm{ppm} & 7.150 \mathrm{ppm} \\ { }^{3} \mathrm{~J}_{\mathrm{AB}}={ }^{3} \mathrm{~J}_{\mathrm{A}^{\prime} \mathrm{B}^{\prime}} & 7.92 \mathrm{~Hz} & 7.92 \mathrm{~Hz} \\ { }^{3} \mathrm{~J}_{\mathrm{BC}}={ }^{3} \mathrm{~J}_{\mathrm{B}^{\prime} \mathrm{C}} & 7.47 \mathrm{~Hz} & 7.47 \mathrm{~Hz} \\ { }^{4} \mathrm{~J}_{\mathrm{AA}^{\prime}} & 2.05 \mathrm{~Hz} & 2.05 \mathrm{~Hz} \\ { }^{4} \mathrm{~J}_{\mathrm{BB}^{\prime}} & 1.42 \mathrm{~Hz} & 1.42 \mathrm{~Hz} \\ { }^{4} \mathrm{~J}_{\mathrm{AC}}={ }^{4} \mathrm{~J}_{\mathrm{A}^{\prime} \mathrm{C}} & 1.22 \mathrm{~Hz} & 1.22 \mathrm{~Hz} \\ { }^{5} \mathrm{~J}_{\mathrm{AB}}={ }^{5} \mathrm{~J}_{\mathrm{A}^{\prime} \mathrm{B}} & 0.60 \mathrm{~Hz} & 0.60 \mathrm{~Hz}\end{array}$

Recorded and analyzed at $400 \mathrm{MHz}$ in $\mathrm{CDCl}_{3}$, but the same dataset can be used to calculate spectra at any magnetic field strength.

Table 2. Chemical Shift difference $(\Delta \delta)$ in $\mathrm{Hz}$ and Ratio with Coupling Constants.

$\begin{array}{ccccc} & \text { Cis-stilbene } & \text { Trans-stilbene } & \text { Cis-stilbene } & \text { Trans-stilbene } \\ \text { Field strength } & 400 \mathrm{MHz} & 400 \mathrm{MHz} & 60 \mathrm{MHz} & 60 \mathrm{MHz} \\ \Delta \delta \text { A - B } & 10.51 \mathrm{~Hz} & 63.42 \mathrm{~Hz} & 1.58 \mathrm{~Hz} & 9.51 \mathrm{~Hz} \\ \Delta \delta \mathrm{B}-\mathrm{C} & 13.74 \mathrm{~Hz} & 39.57 \mathrm{~Hz} & 2.06 \mathrm{~Hz} & 5.94 \mathrm{~Hz} \\ & & & & \\ & & & & 9.513 / 7.92=1.20 \\ \Delta \delta \mathrm{A}-\mathrm{B} /{ }^{3} \mathrm{~J}_{\mathrm{AB}} & 10.51 / 7.92=1.33 & 63.42 / 7.92=8.00 & 1.577 / 7.92=0.20 & 5.936 / 7.47=0.80 \\ \Delta \delta \mathrm{B}-\mathrm{C} /{ }^{3} \mathrm{~J}_{\mathrm{BC}} & 13.74 / 7.47=1.84 & 39.57 / 7.47=5.29 & 2.061 / 7.47=0.28 & 15.45 / 1.22=12.66 \\ & & & 3.64 / 1.22=2.98 & 9.51 / 0.6=15.86 \\ \Delta \delta \mathrm{A}-\mathrm{C} /{ }^{4} \mathrm{~J}_{\mathrm{AC}} & 24.25 / 1.22=19.88 & 102.99 / 1.22=84.42 & 1.58 / 0.6=2.63 & \\ \Delta \delta \text { A }-\mathrm{B}^{\prime} /{ }^{5} \mathrm{~J}_{\mathrm{AB}^{\prime}} & 10.51 / 0.60=17.52 & 63.42 / 0.60=105.7 & & \\ \text { Recorded and analyzed at } 400 \mathrm{MHz} \text { and } 60 \mathrm{MHz} \text { in } \mathrm{CDCl}_{3} . & & \\ \text { For } \Delta \delta / \mathrm{J}<5 \text { then second-order coupling effects are expected. } & \end{array}$

The ${ }^{1} \mathrm{H}$ NMR spectrum of trans-stilbene is comprised of three magnetically-distinct sets of aromatic hydrogen nuclei, together with the vinylic hydrogen, which are labelled $\mathrm{AA}^{\prime}, \mathrm{BB}^{\prime}, \mathrm{C}$ (aromatics) and D (vinylic), as shown in Figure 2. If the ${ }^{3} \mathrm{~J}_{\mathrm{HH}}$ couplings are considered, $(\sim 8.0 \mathrm{~Hz})$ then the expected multiplet patterns would be $\mathrm{AA}^{\prime}$, doublet; $\mathrm{BB}^{\prime}$, triplet; and $\mathrm{C}$, triplet, with the integration ratio of 2:2:1, respectively, for the ortho-, meta- and para-position hydrogens $(2,6-, 3,5-$, and 4-positions). However, it is important to include additional coupling constant parameters in such evaluations, since the magnetic inequivalence of the $\mathrm{A}$ and $\mathrm{A}^{\prime}$, and $\mathrm{B}$ and $\mathrm{B}^{\prime}$ nuclei gives rise to significant couplings between them, i.e. of the order of approximately $1-2 \mathrm{~Hz}$. Once the longrange ${ }^{4} \mathrm{~J}_{\mathrm{AC}},{ }^{4} \mathrm{~J}_{\mathrm{A}^{\prime} \mathrm{C}}$ couplings of $\sim 1.22 \mathrm{~Hz}$ and ${ }^{5} \mathrm{~J}_{\mathrm{AB}^{\prime}},{ }^{5} \mathrm{~J}_{\mathrm{A}^{\prime} \mathrm{B}}$ couplings $\sim 0.6 \mathrm{~Hz}$ are included, then the ${ }^{1} \mathrm{H}$ NMR spectrum can be accurately calculated and predicted at any magnetic field. The chemical shift (ppm) of the $\mathrm{AA}^{\prime}, \mathrm{BB}^{\prime}$ and $\mathrm{C}$ nuclei remain the same at both 400 and $60 \mathrm{MHz}$ magnetic fields 
(indeed any magnetic field). However, the chemical shift differences $\Delta \delta$ in $\mathrm{Hz}$ are significant at 400 and $60 \mathrm{MHz}$ operating frequencies $(400 \mathrm{~Hz}$ per ppm $v$ s $60 \mathrm{~Hz}$ per ppm respectively), and hence spectra recorded at low-field display significant second-order effects, when $\delta \Delta / \mathrm{J}<5$. It is important to note that undergraduates should not be expected to analyze and obtain these values themselves, but employ these data to generate spectra at a variety of magnetic fields in order to observe changes in their appearance and configuration. Analysis of such ${ }^{1} \mathrm{H}$ NMR spectra acquired at $\geq 400$ $\mathrm{MHz}$ provides a dataset of chemical shift and coupling constant values that can then be used for predictively calculating the corresponding $60 \mathrm{MHz}$ spectral profile.

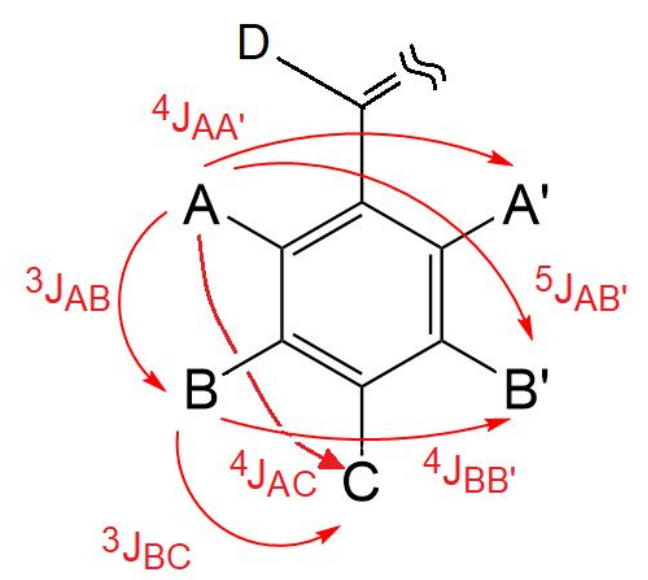

Figure 2. Phenyl moiety and alkene, with the $\mathrm{AA} \mathrm{BB}^{\prime} \mathrm{CD}$ labelling and scalar coupling interactions. 


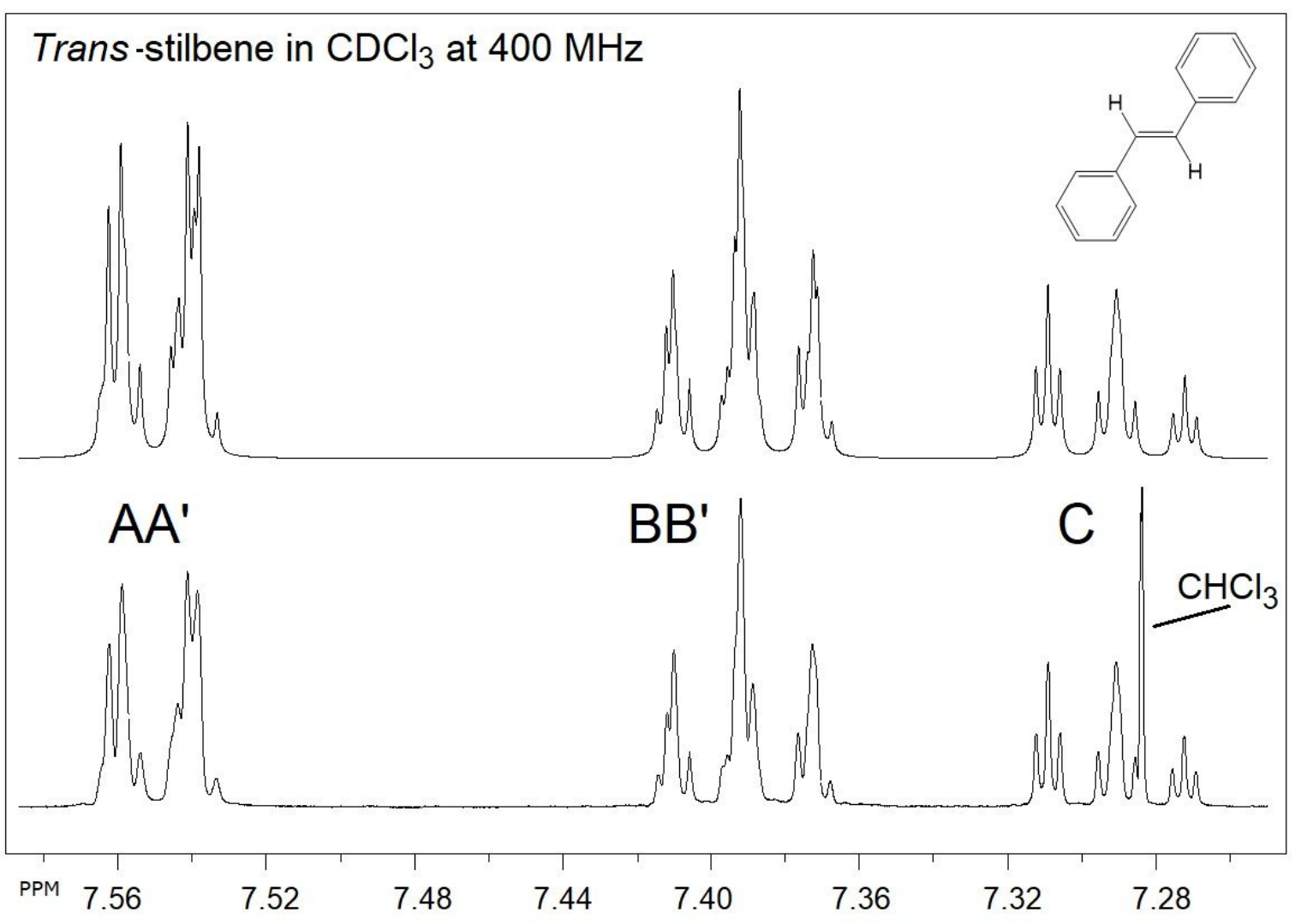

Figure 3. ${ }^{1} \mathrm{H}$ NMR spectrum of trans-stilbene in $\mathrm{CDCl}_{3}$ at $400 \mathrm{MHz}$, displaying the classic double-triplet-triplet patterns. This analysis was performed using ANATOLIA and SpinWorks software programs to yield accurate chemical shift and coupling constants (top: calculated, bottom: experimental). 
Figure 4. ${ }^{1} \mathrm{H}$ NMR spectrum of cis-stilbene in $\mathrm{CDCl}_{3}$ at $400 \mathrm{MHz}$ : Here, second-order effects are significant, and signal overlap and distorted intensities prevent the classic doublet-triplet-triplet patterns from being observed. Nevertheless, computational analysis using ANATOLIA and SpinWorks software programs allowed accurate chemical shift and coupling constant values to be extracted from the profile. (top: calculated, bottom: experimental).

By calculating NMR spectra at a variety of different magnetic field strengths, and comparing these to experimental ones, it is clear that some of the signals that could easily be assigned to "impurities" are actually part of the NMR spectrum. This is clear in Figures 3 and 4, in which the classic doublet-triplet-triplet system is clearly visible in trans-stilbene at $400 \mathrm{MHz}$, but more difficult to visualize for the cis-isomer since the chemical shift values of these aromatic ${ }^{1} \mathrm{H}$ nuclei are very similar and the second-order effects are significant. The calculated NMR spectra for 400$45 \mathrm{MHz}$ of trans-stilbene serves as a useful illustration to students to guide them from familiar high-field NMR spectra to less familiar low-filed NMR spectra. In Figure 5, the cis-stilbene spectrum obtained at only $60 \mathrm{MHz}$ displays low intensity signals both upfield and downfield of the aromatic signal envelope, which may erroneously be attributed to impurities, and the asymmetry of the main aromatic resonance may be explained as "poor shimming". Only by comparing the calculated spectrum to the experimental spectrum can these signals be rationalized unequivocally. 


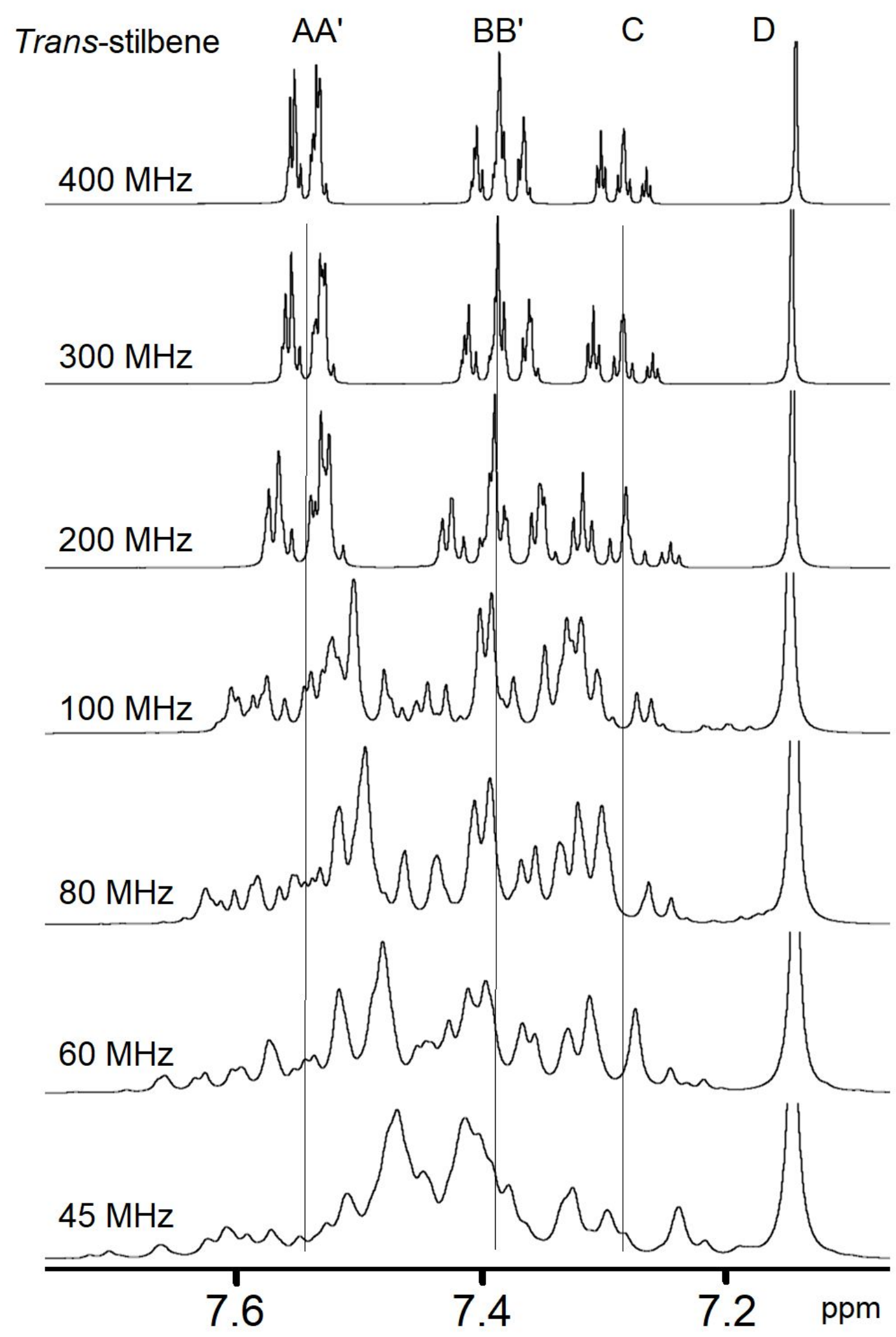

Figure 5. Calculated ${ }^{1} \mathrm{H}$ NMR spectrum of trans-stilbene in $\mathrm{CDCl}_{3}$ at $400,300,200,100,80,60$ and $45 \mathrm{MHz}$ using NMR-SIM software and accurate chemical shift and coupling constant values. Linewidths were kept to a minimum in order to show as much detail as possible. The experimental spectrum at an operating frequency of $60 \mathrm{MHz}$ shows an excellent fit to the calculated profile. 
The Wittig reaction typically yields $\sim 60-70 \%$ of the cis-stilbene product, and $30-40 \%$ of the 245 trans-product, and these two isomers are readily distinguishable in the $60 \mathrm{MHz}{ }^{1} \mathrm{H} \mathrm{NMR}$ spectrum via their vinylic proton signals located at $\delta=6.63 \mathrm{ppm}$ and $7.15 \mathrm{ppm}$ respectively. For this experiment, $\sim 100 \mathrm{mM}$ solutions were used $\left(\sim 20 \mathrm{mg}\right.$ of total stilbene in $0.70 \mathrm{~mL}$ of $\left.\mathrm{CDCl}_{3}\right)$ which provided an excellent signal-to-noise (SNR) ratio of 208 (Bruker TOPSPIN-4.0.3 "sinocal" command ) with only 16 scans completed on the benchtop system. Determination of the exact cis-:trans- ratio of stilbenes synthesized by each student during a practical laboratory class can be readily obtained by integrating the vinylic proton resonances; an example of the appearance of the calculated spectrum for a 70:30 mol\% mixture of these isomers is shown in Figure 6. It is important to highlight that the SNR is of such a high level that an acceptable spectrum can be obtained using a single scan using a $\sim 100 \mathrm{mM}$ solution, and still provides a reliable estimate of the cis-:trans- ratios of stilbene product analytes. Therefore, solutions of $\sim 10 \mathrm{mM}\left(\sim 2 \mathrm{mg}\right.$ sample in $\left.0.70 \mathrm{~mL} \mathrm{CDCl}_{3}\right)$ could be used with a larger number of scans.

With regard to Wittig reaction chemistry, it is clear from these studies that using $\sim 2 \mathrm{~g}$ of the benzaldehyde starting material for this purpose is excessive, and that much smaller amounts could be used. The cost of benzaldehyde is $\sim \$ 35$ for $100 \mathrm{~g}$, and that of benzyltriphenylphosphonium chloride is $\sim \$ 60$ for $100 \mathrm{~g}$ of material. Moreover, there are significant cost savings achievable via reductions in the amounts and volumes of reagents and solvents required, i.e. dichloromethane (20 $\mathrm{mL})$, aqueous $\mathrm{NaOH}$ solution $(20 \mathrm{~mL})$, distilled water $(30 \mathrm{~mL})$, saturated aqueous sodium bisulfite solution $(50 \mathrm{~mL})$, anhydrous sodium sulfate ( $\sim \mathrm{g})$, absolute ethanol $(30 \mathrm{~mL})$, low-b.pt $\left(30-60{ }^{\circ} \mathrm{C}\right)$ petroleum ether $(30 \mathrm{~mL})$, etc.; typical current standard undergraduate laboratory requirement values are provided in brackets. One salient point to highlight is that benchtop NMR spectrometers do not necessitate the use of deuterated solvents, which therefore offers wider choice of low-cost solvents and a significant financial saving, thus a judicious selection of a solvent that has signals in a different part of the spectrum from the signals of interest can yield perfectly acceptable spectra, particularly if solvent suppression methods are employed. Therefore, this novel low-field benchtop NMR analysis approach offers major economic budgetary advantages to undergraduate teaching laboratory activities. 

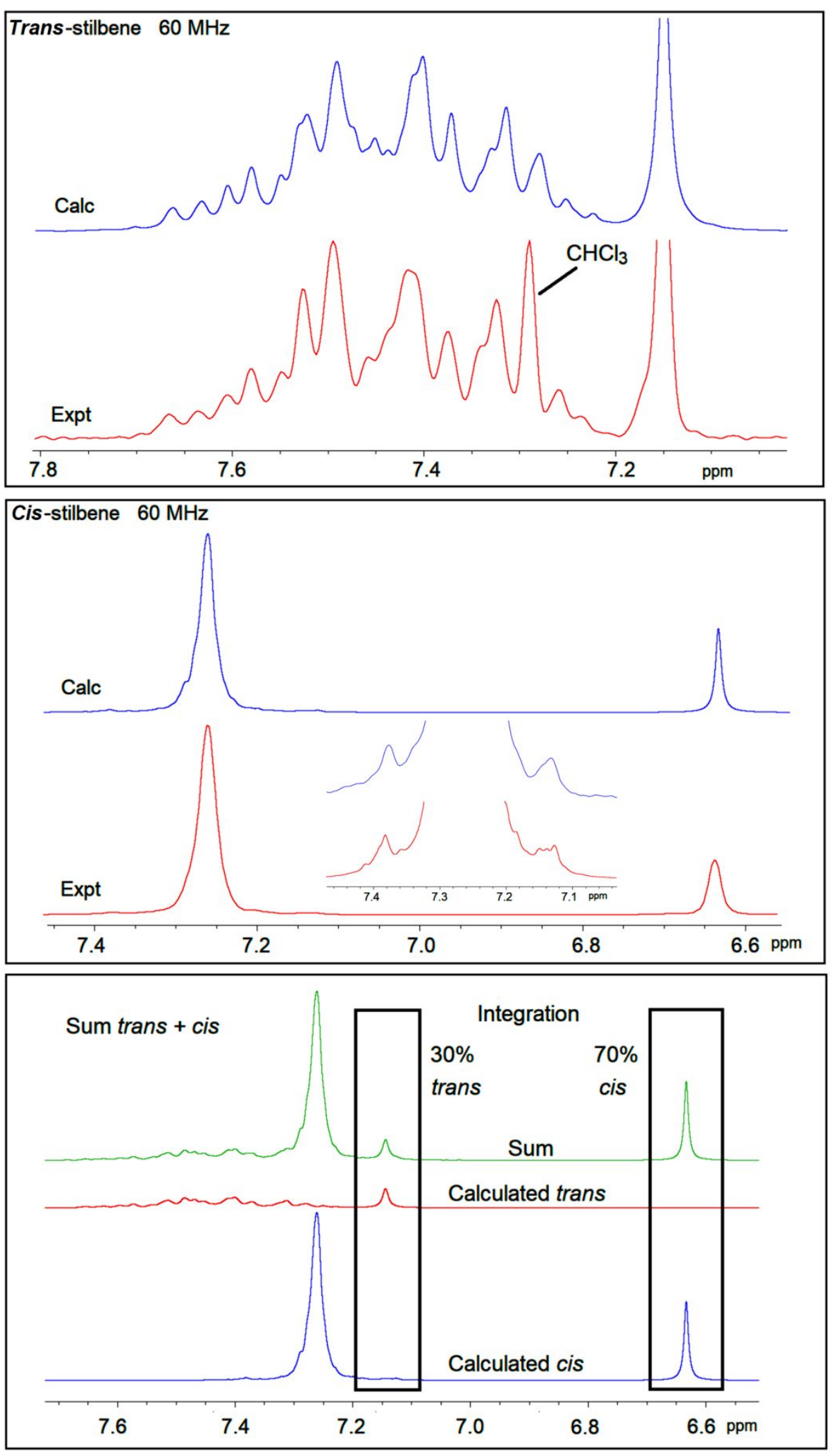

Figure 6. Calculated and experimental ${ }^{1} \mathrm{H}$ NMR spectra of trans-stilbene in $\mathrm{CDCl}_{3}$ at $60 \mathrm{MHz}$ (upper); calculated and experimental ${ }^{1} \mathrm{H}$ NMR spectra of cis-stilbene in $\mathrm{CDCl}_{3}$ at $60 \mathrm{MHz}$ (centre); and calculated ${ }^{1} \mathrm{H}$ NMR spectra of a 70:30 mixture of cis-; trans-stilbene at 60 $\mathrm{MHz}$ (lower). Computational analysis was performed using ANATOLIA, WinDNMR, NMR-SIM and/or SpinWorks software modules to yield accurate chemical shift and coupling constant values. 


\section{EXPERIMENTAL}

The lab experiment is part of the $2^{\text {nd }}$ year undergraduate practical chemistry class and is set up for $\sim 110$ students each year. The undergraduates have access to the lab for 4 hours at a time with up to a maximum of 20 hours available for the experiment - however, this is very rarely required. A single lab session is sufficient for most students to complete the chemistry. Academics create the lab classes, technical tutors (with degree and $\mathrm{PhD}$ level qualifications) run the lab class, post-docs and $\mathrm{PhD}$ students monitor the lab classes. Group demonstrations are provided for "tricky" chemistry at the start of the class, thereafter the students follow a detailed lab script. Careful reagent handling is required throughout the lab class, particularly for the handling of $\mathrm{NaOH}$ solution and starting materials. The lab capacity of $\sim 60$ requires that the year group is split in two and the class ran twice. The students are not expected to use the ANATOLIA software, but have access to benchtop NMR spectrometers and handouts of $400 \mathrm{MHz}$ NMR spectra which connects the $400 \mathrm{MHz}$ result to the $40-80 \mathrm{MHz}$ results.

The second-year students have 12 NMR lectures very early in the term, covering spin, chemical shift, couplings, 13C-DEPT, COSY and HMQC, nOe, $\mathrm{T}_{1}$ and $\mathrm{T}_{2}$, solvent and temperature effects, inorganic NMR, solid-state NMR. The second year students have an assessed NMR workshop question ( $\mathrm{H}, \mathrm{C}, \mathrm{DEPT}, \mathrm{COSY}, \mathrm{HMQC}$ and nOe) in term 1 where then need to assign ${ }^{1} \mathrm{H}$ and ${ }^{13} \mathrm{C}$ signals, this year they have cinnamyl acetate, and construct multiplets and the contributing ${ }^{1} \mathrm{H}-{ }^{1} \mathrm{H}$ interactions from neighbors.

High-field ${ }^{1} \mathrm{H}$ spectra were recorded using JEOL ECS-400 MHz or Bruker Avance-I $400 \mathrm{MHz}$ NMR spectrometers, using an auto-tune probe and $5 \mathrm{~mm}$ NMR tubes. Samples were prepared using 20 mg of stilbene dissolved in a $0.70 \mathrm{~mL}$ volume of $\mathrm{CDCl}_{3}$ obtained from Apollo-UK, i.e. $100 \mathrm{mM}$ solutions. High quality 5-mm diameter NMR tubes purchased from Norell were used at both 400 and $60 \mathrm{MHz}$ operating frequencies. ${ }^{1} \mathrm{H}$ NMR spectra were recorded using the small flipangle pulse program with $\mathrm{P}_{90}=15.25 \mu$ s covering a sweep width of $12.0 \mathrm{ppm}(4,789 \mathrm{~Hz})$ with $64 \mathrm{~K}$ time domain data points giving an acquisition time of $685 \mathrm{~s}$, with a relaxation delay of $5 \mathrm{~s}$, Fouriertransformed using $128 \mathrm{~K}$ data points and referenced to an internal TMS standard at $0.00 \mathrm{ppm}$. Benchtop ${ }^{1} \mathrm{H}$ NMR spectra were acquired on a Magritek-Ultra $60 \mathrm{MHz}$ NMR spectrometer, with 16 scans, covering a sweep-width of $81 \mathrm{ppm}$ with $64 \mathrm{~K}$ data points in the FID, and providing an 
acquisition time of $6.55 \mathrm{~s}$ and a digital resolution of $0.059 \mathrm{~Hz}$ (pulse angle was $90^{\circ}$ with a pulse

length of $12.8 \mu \mathrm{s})$. FID fourier transformed using zero-filling to $128 \mathrm{k}$ data points providing a spectrum with $0.038 \mathrm{~Hz}$ resolution. The $\mathrm{T}_{1}$ relaxation time was measured and found to be under 2 $\mathrm{s}$ for each signal, and therefore a repetition time of $10 \mathrm{~s}$ between scans was sufficient to allow relaxation of the ${ }^{1} \mathrm{H}$ nuclei to equilibrium subsequent to each scan. The total experimental time required to record the ${ }^{1} \mathrm{H}$ NMR spectrum was $2 \min$ and $40 \mathrm{~s}$. The temperature of the sample at both 400 and $60 \mathrm{MHz}$ was $20^{\circ} \mathrm{C}$ (the magnet temperature was $26.5^{\circ} \mathrm{C}$ for the latter spectrometer).

Following the reaction of benzaldehyde with benzyltriphenylphosphonium chloride (typically 2.0 $\mathrm{g}$ and $7.4 \mathrm{~g}$ respectively, equivalent to a 1:1 molar ratio) in dichloromethane $(20 \mathrm{~mL})$, products form on addition of $20 \mathrm{~mL}$ of aqueous $\mathrm{NaOH}$ solution $(50 \% \mathrm{w} / \mathrm{w})$, the mixture being stirred for 30 min. Subsequently, the organic phase was separated, washed with a $30 \mathrm{~mL}$ volume of distilled water, and then $50 \mathrm{~mL}$ of a saturated solution of sodium bisulfite was added until the solution was neutralized. The organic phase was then dried over anhydrous sodium sulfate, filtered and evaporated to dryness. Finally, $30 \mathrm{~mL}$ of absolute ethanol was added to the thick cloudy residue, and the mixture then cooled in an ice bath for $15 \mathrm{~min}$. The primary precipitate obtained was transstilbene (yield ca.1.0 g), mp $122-123^{\circ} \mathrm{C}$. The filtrate was then evaporated, and a $40 \mathrm{~mL}$ volume of low boiling-point petroleum ether $\left(30-60^{\circ} \mathrm{C}\right)$ was added to precipitate triphenylphosphine oxide $(\sim 5$ g), $\mathrm{mp} 146-147^{\circ} \mathrm{C}$. The filtrate arising following removal of triphenylphosphine oxide was then evaporated, yielding a liquid, cis-stilbene (yield $\sim 1.5 \mathrm{~g}$ ).

\section{HAZARDS}

Benzyltriphenylphosphoniumchloride (CAS Number 1100-88-5) can be fatal if swallowed or if inhaled, it is toxic in contact with skin, causes skin irritation, causes serious eye irritation, may cause respiratory irritation, toxic to aquatic life with long lasting effects. Dispose of in halogen waste.

Benzaldehyde (CAS Number 100-52-7), harmful if swallowed, harmful in contact with skin, causes skin irritation, Dispose of in hydrophilic waste.

Sodium hydroxide (CAS 1310-73-2) may be corrosive to metals, causes severe skin burns and eye damage, neutralised with $5 \mathrm{M} \mathrm{HCl}$. 
Magnesium sulfate (CAS Number 7487-88-9), no hazard statements applicable dispose of in waste bin.

Dichloromethane (CAS Number 75-09-2), causes skin irritation, causes serious eye irritation, may cause respiratory irritation, may cause drowsiness or dizziness, suspected of causing cancer, may cause damage to organs. Dispose of in halogenated waste.

Sodium bi-sulfite saturated solution (CAS Number 7631-90-5), harmful if swallowed, causes serious eye damage, contact with acids liberates toxic gas.bi-sulfite waste bottle provided. Ethanol (CAS Number 64-17-5), highly flammable liquid and vapor, causes serious eye irritation, hydrophilic waste, light petroleum highly flammable liquid and vapor, harmful if swallowed, may be fatal if swallowed and enters airways, causes skin irritation, may cause drowsiness or dizziness, toxic to aquatic life with long lasting effects. Dispose of in hydrophobic waste.

(E)- (Z)- stilbene product (trans- CAS Number 103-30-0) (cis- CAS Number 645-49-8), harmful if swallowed, causes serious eye irritation, toxic to aquatic life with long lasting effects.

Triphenylphosphine oxide (CAS Number 791-28-6) product harmful if swallowed, causes skin irritation, causes serious eye irritation, may cause respiratory irritation,

\section{CONCLUSIONS}

A combination of 'state-of-the-art' spectral analysis and experimental benchtop NMR methods provides valuable information which helps to explain of the overlapping features that are common in low-field NMR spectra. This renders benchtop NMR spectrometer systems much more applicable and accessible to undergraduate teaching laboratories. One significant advantage of calculating low-field NMR spectra is that commonly-employed undergraduate laboratory experiments in synthetic organic chemistry or other areas which may be dismissed as being "too complicated" or "lacking sufficient analytical information" can now be understood and used, without recourse to wholesale changes in methods or reagents, which may have unwanted cost and safety implications. In conclusion, ANATOLIA software is an effective and robust tool which markedly facilitated the extraction of chemical shift and scalar coupling constant values from second-order overlapping spectra. 


\section{ASSOCIATED CONTENT}

Supporting Information

Supporting_Info:

The Supporting Information is available on the ACS Publications website at DOI:

10.1021/acs.jchemed.XXXXXXX. [ACS will fill this in.]

Introduction to help install the ANATOLIA program, ABX spin-system example, description of the ANATOLIA input data files, $\mathrm{ABX}$ output file, input data files for stilbene, $400 \mathrm{MHz}{ }^{1} \mathrm{H}$ NMR spectrum of trans-stilbene, $400 \mathrm{MHz}{ }^{1} \mathrm{H}$ NMR spectrum of cis-stilbene, $60 \mathrm{MHz}, 80 \mathrm{MHz}$ and 400 $\mathrm{MHz}{ }^{1} \mathrm{H}$ NMR spectra of crude reaction mixture (triphenylphosphine oxide, trans-stilbene and cisstilbene (DOCX).

\section{AUTHOR INFORMATION \\ Corresponding Author \\ *E-mail: m.edgar@1boro.ac.uk \\ ORCID Mark Edgar: 0000-0002-8579-1088 \\ ResearcherID: V-4166-2017}

The authors declare no completing financial interest.

\section{ACKNOWLEDGMENTS}

We acknowledge Magritek (Philipsstraße 8, 52068 Aachen, Germany) for the loan of a SpinSolve-60 Ultra benchtop NMR spectrometer to the School of Pharmacy (DMU) and for $80 \mathrm{MHz}$ NMR spectra of the crude mixture of cis- trans-stilbene, Ben Buckley's research group (University of Loughborough) for pure samples of cis- and trans-stilbenes, and finally Stuart Pinkney for computational and IT expertise.

\section{REFERENCES}

(1) Wittig, G.; Geissler, G. Zur Reaktionsweise des Pentaphenyl-phosphors und einiger Derivate. Justus Liebigs Ann. Chem., 1953, 580, 44. doi.org/10.1002/jlac. 19535800107 
(2) Markl, G.; Merz, A., Carbonyl-Olefinierungen mit nicht-stabilisierten Phosphinalkylenen im wäßrigen System. Synthesis, 1973, 5, 295-297. doi.org/10.1055/s1973-22193

(3) Warner, J. C.; Anastas, P. T.; Anselme, J. P. The Wittig reaction in the undergraduate organic laboratory. J Chem. Educ, 1985, 62 (4), 346. DOI: $10.1021 /$ ed062p346

(4) Mills, N. S.; Shanklin, M., Access to NMR Spectroscopy for Two-Year College Students: The NMR Site at Trinity University. J. Chem. Educ., 2011, 88 (6), 835-839. DOI: $10.1021 /$ ed $100715 y$

(5) Edgar, M. Physical Methods and Techniques: NMR Spectroscopy. Annu. Rep. Prog. Chem., Sect. B, 2011, 107, 308-327. DOI: 10.1039/C1OC90006D

(6) Edgar, M. Physical Methods and Techniques: NMR Spectroscopy. Annu. Rep. Prog. Chem., Sect. B, 2013, 109, 256-274. DOI: 10.1039/C3OC90012F

(7) Grootveld, M.; Percival, B. C.; Gibson, M.; Osman, Y.; Edgar, M.; Molinari, M.; Mather, M. L.; Casanova, F.; Wilson, P. B. Progress in low-field benchtop NMR spectroscopy in chemical and biochemical analysis, Analytica Chimica Acta, Available online 23 February 2019. https://doi.org/10.1016/

(8) Percival, B. C.; Grootveld, M.; Gibson, M.; Osman, Y.; Molinari, M.; Jafar, F.; Sahota, T.; Martin, M.; Casanova, F.; Mather, M. L.; Edgar, M.; Masania, J.; Wilson, P. B. Low-Field, Benchtop NMR Spectroscopy as a Potential Tool for Point-of-Care Diagnostics of Metabolic Conditions: Validation, Protocols and Computational Models. High-Throughput, 2019, 8(1), 2-33. https://doi.org/10.3390/ht8010002.

(9) Reisch, M. S. An NMR Renaissance. Chem. Eng. News, 2015, 93 (37), 19-21. DOI: 10.1021/cen-09337-bus 1

(10) Soulsby, D.; Wallner, A.S. Introduction to NMR Spectroscopy in the Undergraduate Curriculum, NMR Spectroscopy in the Undergraduate Curriculum: First Year and Organic Chemistry Courses Volume 2, Chapter 1, pp 1-10, ACS Symposium Series, Vol. 1221, 2016. DOI: 10.1021/bk-2016-1221.ch001

(11) Dimick Gray, S. M. Using Benchtop NMR in Undergraduate Organic Courses, NMR Spectroscopy in the Undergraduate Curriculum: First Year and Organic Chemistry Courses Volume 2, Chapter 8, pp 107-118, ACS Symposium Series, Vol. 1221, 2016. DOI: $10.1021 / \mathrm{bk}-2016-1221 . \mathrm{ch} 008$

(12) Masania, J.; Grootveld, M.; Wilson, P. B. Teaching Analytical Chemistry to Pharmacy Students: A Combined, Iterative Approach. J. Chem. Educ., 2018, 95, 47-54. DOI: 10.1021/acs.jchemed.7b00495 
(13) Yearty, K. L.; Sharp, J. T.; Meehan, E. K.; Wallace, D. R., Jackson, D. M.; Morrison R. W. Implementation of PicoSpin Benchtop NMR Instruments into Organic Chemistry Teaching Laboratories through Spectral Analysis of Fischer Esterification Products. J. Chem. Educ. 2017, 94 (7), 932-935. DOI: $10.1021 /$ acs.jchemed.6b00972

(14) Isaac-Lam, M. F. Analysis of Bromination of Ethylbenzene Using a $45 \mathrm{MHz} \mathrm{NMR}$ Spectrometer: An Undergraduate Organic Chemistry Laboratory Experiment. $J$. Chem. Educ. 2014, 91 (8), 1264-1266. DOI: 10.1021/ed400365p

(15) Bonjour, J. L.; Hass, A. L.; Pollock, D. W.; Huebner, A; Frost J. A. Bringing NMR and IR Spectroscopy to High Schools. J. Chem. Educ. 2017, 94 (1), 38-43. DOI: $10.1021 /$ acs.jchemed.6b00406

(16) https://www.stem.org.uk/ (accessed March 2019).

(17) Mann, B. E. The Analysis of First-Order Coupling Patterns in NMR Spectra. J. Chem. Educ., 1995, 72 (7), 614-615. DOI: 10.1021/ed072p614

(18) Abraham, R. J. The Analysis of High Resolution NMR Spectra, Elsevier Publishing Co., New York, 1971. DOI: 10.1021/ed049pA603.

(19) Levitt, M. H. Spin Dynamics, basics of nuclear magnetic resonance, John Wiley \& sons, p211, 2001, ISBN: 0471489220

(20) Richards, R. E.; Schaeffer, T. P. High Resolution Proton Resonance Spectra of some p-substituted Benzenes. Trans. Faraday Soc. 1958, 54, 1280. DOI: $10.1039 /$ TF9585401280

(21) Corio, P. L.; Structure of High-Resolution NMR-Spectra, Academic Press. New York 1966, 143. https://doi.org/10.1002/bbpc.19670710722

(22) Gunther, H. 1H-NMR Spectra of the $\mathrm{AA}^{\prime} \mathrm{XX}^{\prime}$ and $\mathrm{AA}^{\prime} \mathrm{BB}^{\prime}$ Type - Analysis and Classification. Angew. Chem. Int. Ed. 1972, 11 (10), 861-873. doi.org/10.1002/anie.197208611.

(23) Abraham R. J.; Bernstein H. J. The Proton Magnetic Resonance Spectra of Thiophene. Can. J. Chem. 1959, 37, 2095-2097. https://doi.org/10.1139/v59-307

(24) Abraham R. J.; Bernstein H. J. The Proton Resonance Spectra of Furan and Pyrrole. Can. J. Chem. 1959, 37, 1056-1065. https://doi.org/10.1139/v59-153

(25) Gutowsky H. S., Holm, C.H., Saika, A., and Williams, G. A. Electron Coupling of Nuclear Spins. I. Proton and Fluorine Magnetic Resonance Spectra of Some Substituted Benzenes. J. Am. Chem. Soc. 1957, 79 (17), 4596. DOI: $10.1021 / \mathrm{ja0} 1574 \mathrm{a} 007$ 
(26) Edgar W. Garbisch Jr., Analysis of complex NMR spectra for the organic chemist. I. Second-order approach with specific application to the two spin system, J. Chem. Educ. 1968, 45 (5), 311-321. DOI: 10.1021/ed045p311

(27) Edgar W. Garbisch Jr., Analysis of complex NMR spectra for the organic chemist. II. Three spin systems of the ABC, ABX, ABK, and AB2 types, J. Chem. Educ. 1968, 45 (6), 402-416. DOI: 10.1021/ed045p402

(28) Edgar W. Garbisch Jr., Analysis of complex NMR spectra for the organic chemist: III. Four spin systems of the $\mathrm{ABC} 2, \mathrm{ABX} 2, \mathrm{ABK} 2, \mathrm{AA}^{\prime} \mathrm{BB}^{\prime}$, and $\mathrm{AA}^{\prime} \mathrm{XX}$ ' types, J. Chem. Educ. 1968, 45 (7), 480-493. DOI: 10.1021/ed045p480

(29) Clark, M.; Thrasher, J. S. LAOCOON PC: NMR simulation on a personal computer, J. Chem. Educ., 1990, 67 (3), 235. DOI: 10.1021/ed067p235.2

(30) Bruker software available in1970s onwards on the ASPECT computer.

(31) Reich, H. J. WinDNMR: Dynamic NMR Spectra for Windows. J. Chem. Educ., 1995, 72 (12), 1086. DOI: 10.1021/ed072p1086.1

(32) NMRSIM Copyright (C) 1999 by Bruker Analytik GmbH. Part No. H9171.

(33) SpinWorks 3.1, Copyright (C) 2009, Kirk Marat, University of Manitoba.

(34) Martin, J. S.; Quirt, A. R. NMR Spectra of Symmetric Molecules. I. The Spin Hamiltonian for Twofold Symmetry, J. Magn. Reson. 1971, 5, 318-327. https://doi.org/10.1016/0022-2364(71)90082-5

(35) Cheshkov, D. A.; Sheberstov, K. F.; Sinitsyn, D. O.; Chertkov, V. A. ANATOLIA: NMR software for spectral analysis of total line-shape. Magn. Reson. Chem., 2018, 56, 449-457. doi: 10.1002/mrc.4689.

(36) Gaussian 16, Revision B.01, Frisch, M. J.; Trucks, G. W.; Schlegel, H. B.; Scuseria, G. E.; Robb, M. A.; Cheeseman, J. R.; Scalmani, G.; Barone, V.; Petersson, G. A.; Nakatsuji, H.; Li, X.; Caricato, M.; Marenich, A. V.; Bloino, J.; Janesko, B. G.; Gomperts, R.; Mennucci, B.; Hratchian, H. P.; Ortiz, J. V.; Izmaylov, A. F.; Sonnenberg, J. L.; Williams-Young, D.; Ding, F.; Lipparini, F.; Egidi, F.; Goings, J.; Peng, B.; Petrone, A.; Henderson, T.; Ranasinghe, D.; Zakrzewski, V. G.; Gao, J.; Rega, N.; Zheng, G.; Liang, W.; Hada, M.; Ehara, M.; Toyota, K.; Fukuda, R.; Hasegawa, J.; Ishida, M.; Nakajima, T.; Honda, Y.; Kitao, O.; Nakai, H.; Vreven, T.; Throssell, K.; Montgomery, J. A., Jr.; Peralta, J. E.; Ogliaro, F.; Bearpark, M. J.; Heyd, J. J.; Brothers, E. N.; Kudin, K. N.; Staroverov, V. N.; Keith, T. A.; Kobayashi, R.; Normand, J.; Raghavachari, K.; Rendell, A. P.; Burant, J. C.; Iyengar, S. S.; Tomasi, J.; Cossi, M.; Millam, J. M.; Klene, M.; Adamo, C.; Cammi, R.; Ochterski, J. 
W.; Martin, R. L.; Morokuma, K.; Farkas, O.; Foresman, J. B.; Fox, D. J. Gaussian, Inc., Wallingford CT, 2016.

(37) Abraham, R. J.; Edgar, M.; Griffiths, L.; Powell, R. L. Calculation of Proton ChemicalShifts in Hydrocarbons. J. Chem. Soc., Chem. Commun, 1993, 20, 1544-1545. DOI: $10.1039 / \mathrm{C} 39930001544$

(38) Abraham, R. J.; Edgar, M.; Griffiths, L.; Powell, R. L. Substituent chemical shifts (SCS) in NMR. Part 5. Mono-and di-fluoro SCS in rigid molecules. J. Chem. Soc., Perkin Trans. 2, 1995, 24, 561-567. DOI: 10.1039/P29950000561

(39) http://mestrelab.com/software/mnova/nmr/ (accessed March 2019).

(40) Weber U.; Spiske R.; Hoffken H. W.; Haigele G.; Thiele H., WIN-DAISY Manual. Bremen. Bruker-Franzen Analytik GmbH 1993. 